\title{
Absence of detection of RSV and influenza during the COVID-19 pandemic in a Brazilian cohort: Likely role of lower transmission in the community
}

Fernanda Hammes Varela1,2*, Marcelo Comerlato Scotta ${ }^{1,2 *}$, Márcia PoleseBonatto ${ }^{1}$, Ivaine Tais Sauthier Sartor ${ }^{1}$, Charles Francisco Ferreira', Ingrid Rodrigues Fernandes ${ }^{1}$, Gabriela Oliveira Zavaglia ${ }^{1}$, Walquiria Aparecida Ferreira de Almeida ${ }^{3}$, Denise Arakaki-Sanchez ${ }^{4}$, Leonardo Araújo Pinto², Gisele Alsina Nader Bastos ${ }^{1}$, Luiz Antônio Nasi ${ }^{1}$, Maicon Falavigna ${ }^{1}$, Paulo Márcio Pitrez ${ }^{1}$, Renato T Stein ${ }^{1,2}$; COVIDa study group

${ }^{1}$ Social Responsibility - PROADI-SUS, Hospital Moinhos de Vento, Porto Alegre, Brazil ${ }^{2}$ School of Medicine, Pontifícia Universidade Católica do Rio Grande do Sul, Porto Alegre, Brazil ${ }^{3}$ General Coordination, Programa Nacional de Imunização, Brazilian Ministry of Health, Brasilia, Brazil

${ }^{4}$ General Coordination, Programa de Vigilância das Doenças de Transmissão Respiratória de Condições Crônicas, Brazilian Ministry of Health, Brasilia, Brazil

\section{Correspondence to:}

Renato T. Stein

Ramiro Barcelos street, 630/1002

90035-001

Porto Alegre

Brazil

renatotstein@gmail.com

Fernanda Hammes Varela

Ramiro Barcelos, 630/1002

90035-001

Porto Alegre

Brazil

fernanda.varela@hmv.org.br
Background Respiratory syncytial virus (RSV) and influenza are prevalent seasonal community viruses. Although not completely understood, SARS-CoV-2 may have the same means of transmission. Preventive social measures aimed at preventing SARS-CoV-2 spread could impact transmission of other respiratory viruses as well. The aim of this study is to report the detection of RSV and influenza during the period of social distancing due to COVID-19 pandemic in a heavily affected community.

Methods Prospective study with pediatric and adult populations seeking care for COVID-19-like symptoms during the fall and winter of 2020 at two hospitals in Southern Brazil. RT-PCR tests for SARS-CoV-2, influenza A (Flu A), influenza B (Flu B) and respiratory syncytial virus (RSV) was performed for all participants.

Results 1435 suspected COVID-19 participants (1137 adults, and 298 children). were included between May and August. Median age was 37.7 years $(\mathrm{IQR}=29.6-47.7)$, and 4.92 years $(\mathrm{IQR}=1.96$ 9.53), for the adult and child cohorts, respectively. SARS-CoV-2 was positive in 469 (32.7\%) while influenza and RSV were not detected at all.

Conclusions Measures to reduce SARS-CoV-2 transmission likely exerted a huge impact in the spread of alternate respiratory pathogens. These findings contribute to the knowledge about the dynamics of virus spread. Further, it may be considered for guiding therapeutic choices for these other viruses.

Since December 2019 the world experienced the outbreak of the severe acute respiratory syndrome coronavirus 2 (SARS-CoV-2). SARS$\mathrm{CoV}-2$ has spread throughout the world with a catastrophic impact not only on public health but also with significant social and economic burden. Brazil is currently the third most affected country, with 9447165 confirmed diagnosis of COVID-19 and 230034 related deaths up to February 7, 2021 [1].

The response to the pandemic has triggered a major change in overall human behavior, with social distancing measures, teleworking, closing of schools and daycare facilities, closing of businesses, strict hygiene 
behaviors, widespread use of face masks, travel restrictions, and avoidance of activities associated with population gatherings. Brazil has not been an exception to this new scenario, and while it took a while for efficient measures of social distancing to take place, schools and daycare facilities have been closed since mid-March and children have been home since then [2].

As respiratory viruses such as influenza, respiratory syncytial virus (RSV) and SARS-CoV-2 share similar routes and means of transmission, these huge social efforts to prevent the spread of SARS-CoV-2 are also likely to affect the epidemiology of influenza and RSV [3]. Both RSV and influenza have very typical and significant seasonal epidemiology in Brazil, especially during autumn and winter in subtropical areas in the South of the country. An initial concern of health authorities worldwide was the burden of these concomitant infections during the winter months and many clinical guidelines reflected this by indicating both prevention of RSV and treatment of influenza for suspected cases [4,5].

The aim of this study was to report influenza and RSV diagnosis during the SARS-CoV-2 pandemic in pediatric and adult populations with suggestive symptoms of COVID-19 in two health care facilities that serve communities with very different socio-economic backgrounds in the city of Porto Alegre, during local well-defined influenza and RSV seasons [5].

\section{METHODS}

This prospective study was conducted from May 13 to August 31, 2020, two general hospitals (one private and one public) in Porto Alegre, Southern Brazil, a city whose estimated population was 1488252 inhabitants in 2020 [6]. Consecutive adults (>18 year-old) or children older than 2 months were included if presenting at either the outpatient clinics (OPC), emergency departments (ER), or hospitalized with at least one of the following signs or symptoms within 14 days of onset: cough, fever, or sore throat. Exclusion criteria included failure in SARS-CoV-2 sample collection. Clinical and demographic data, as well as samples for viruses testing were collected by a trained research staff at enrollment, according to a standardized protocol.

RT-PCR tests were performed for SARS-CoV-2, influenza A (Flu A), influenza B (Flu B) and RSV for all included subjects. For SARS-CoV-2 analysis nasopharyngeal and oropharyngeal swabs were collected, both swabs allocated in the same transport media. The qualitative Real Time PCR (RT-PCR), was performed with TaqPathTM 1-Step RT-qPCR Master Mix, CG, (Catalog Numbers A15299, AppliedBiosystems, ThermoFisher Scientific, Frederick, USA) and TaqMan ${ }^{\mathrm{TM}}$ 2019-nCoV Assay Kit vl (Catalog Number A47532, ThermoFisher Scientific, Pleasanton, USA), the TaqMan ${ }^{\mathrm{TM}}$ 2019-nCoV Control Kit v1 (Catalog Number A47533, ThermoFisher Scientific, Pleasanton, USA) as reaction control. QuantStudio 5 (ThermoFisher Scientific, Waltham, USA) was used to perform protein chain reaction. For RSV, Flu A and Flu B analysis, material from both nostrils were collected with the Xpert ${ }^{\circledR N a s a l ~ S a m p l e ~ C o l l e c t i o n ~ S w a b ~ B / F-100 ~(C e p h e i d, ~ S u n n y v a l e, ~ U S A) . ~ a n d ~ a l l o c a t e d ~ i n ~ p r o p e r ~}$ transport media (Cepheid, Sunnyvale, USA). Samples were transferred and analyzed in Xpert ${ }^{\circledR X}$ Xpress Flu/RSV cartridges (Cepheid, Sunnyvale, USA) [7].

For the statistical analyses, proportions of specimens positive for SARS-CoV-2, influenza, and RSV were stratified by age (ie, children or adults). Normally distributed quantitative data, according to the Shapiro-Wilk test, were expressed as mean \pm standard deviation $(\mathrm{SD})$, and non-normally distributed quantitative data were expressed by median and interquartile range (IQR). Categorical variables were described as absolute (n) and relative (\%) frequencies.

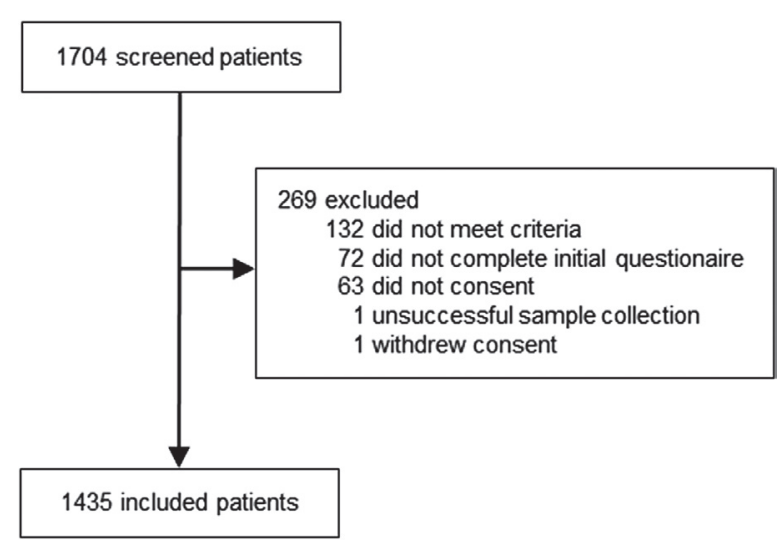

Figure 1. Flow of the participants in the study.
The study protocol was approved by the Institution's ethics review board. Written consent was obtained from all participants and/or legal representatives. The study was conducted according to good laboratory practices and in accordance with the Declaration of Helsinki.

\section{RESULTS}

A total of 1435 participants were included in the study from May 13 to August 31, 2020. Of the 1704 screened subjects, 269 were excluded (132 for not meeting inclusion criteria, 63 for not consenting, 72 for not completing initial questionnaire, 1 unsuccessful sample collection, and 1 withdrew consent), as shown in Figure 1. Median age for adults was 37.8 years (IQR $=29.6-47.8$, 
Table 1. Clinical, demographic and laboratory characteristics

\begin{tabular}{|c|c|c|}
\hline VARIABLE & CHILDREN N = 298 & ADULT N = 1137 \\
\hline Age (years) - median (IQR) & $4.92(1.97-9.51)$ & $37.79(29.61-47.77)$ \\
\hline Female - n (n\%) & $157(52.7)$ & $688(60.5)$ \\
\hline OSS to inclusion (days) - median (IQR) & $3.00(2.00-4.00)$ & $3.00(2.00-5.00)$ \\
\hline \multicolumn{3}{|l|}{ Symptoms - n (n\%): } \\
\hline Fever & $216(72.5)$ & $608(53.5)$ \\
\hline Cough & $221(74.2)$ & $891(78.4)$ \\
\hline Loss of smell/taste sensation & $157(52.7)$ & $649(57.1)$ \\
\hline Shortness of breath & $118(39.6)$ & $479(42.1)$ \\
\hline Sore throat & $113(37.9)$ & $738(64.9)$ \\
\hline Headache & $131(44.0)$ & $957(84.2)$ \\
\hline Nausea/vomiting & $97(32.6)$ & $434(38.2)$ \\
\hline Diarrhea & $73(24.5)$ & $376(33.1)$ \\
\hline Conjunctivitis & $49(16.4)$ & $385(33.9)$ \\
\hline Fatigue & $123(41.3)$ & $853(75.0)$ \\
\hline Muscle/joint pain & $68(22.8)$ & $789(69.4)$ \\
\hline Chills & $74(24.8)$ & $648(57.0)$ \\
\hline \multicolumn{3}{|l|}{ Comorbidities - n (n\%): } \\
\hline Systemic arterial hypertension & $1(0.3)$ & $190(16.7)$ \\
\hline Diabetes mellitus & $1(0.3)$ & $59(5.2)$ \\
\hline Ischemic heart disease & $0(0.0)$ & $17(1.5)$ \\
\hline Cardiac insufficiency & $0(0.0)$ & $18(1.6)$ \\
\hline Chronic obstructive pulmonary disease & $3(1.0)$ & $26(2.3)$ \\
\hline Asthma & $52(17.4)$ & $87(7.7)$ \\
\hline Others & $7(2.3)$ & $21(1.8)$ \\
\hline \multicolumn{3}{|l|}{ Laboratory diagnosis: } \\
\hline SARS-CoV-2 & $49(16.4)$ & $420(36.9)$ \\
\hline influenza A & $0(0.0)$ & $0(0.0)$ \\
\hline influenza B & $0(0.0)$ & $0(0.0)$ \\
\hline Respiratory syncytial virus & $0(0.0)$ & $0(0.0)$ \\
\hline
\end{tabular}

In USA, Asia and Europe, a number of public health measures aiming to prevent the rapid spread of COVID-19 have started mostly at the end of the winter season. The findings of concomitant viral infections in these communities may have been biased by a natural decline in the incidence of both RSV and influenza, although an unprecedented drop in hospitalization due to RSV has been recently described in Alaska [8]. Wu et al also describe that during the COVID-19 pandemic in China there was a decreasing trend in influenza reports early in 2020, in contrast with two spike waves observed in the previous year [9]. In Switzerland, SARS-CoV-2 almost, and very quickly, completely replaced the seasonally circulating community-acquired respiratory viruses within 3 weeks' into the pandemic [10]. This finding raises the hypothesis of a possible competition pattern among respiratory viruses. Yet, some reports of substantial rates of viral coinfection make this explanation unlikely [11].

Another possible explanation for lower influenza rates could be heightened awareness due the pandemic, with a subsequent increase in influenza vaccination numbers. However, in Brazil, influenza vaccination rates were 88.8\% for the target population, similar to historical values [12]. Furthermore, according to nationwide data from the Brazilian Ministry of Health, from March to August-2020, 643090 cases diagnosed with severe lower respiratory tract illness (sLRTI) were notified. Among these $52.2 \%(\mathrm{n}=335748)$ were positive for SARS-CoV-2 and only $0.4 \%$ (2351) for influenza. Similarly, in our region, including Porto Alegre, there were 26508 notifications of sLRTI; from those $12717(48 \%)$ were due to COVID-19 and only $43(0.16 \%)$ were positive for influenza for the same time period [13]. These findings are in stark contrast with 2019 data, where the total number of sLRTI cases associated with influenza was 5800 for the whole of Brazil and 388 in our region, considering the same period of time (unpublished data).

The pattern of RSV was similar, with a significant reduction in the number of hospitalized cases with acute viral bronchiolitis throughout the whole country. In our region hospital admissions for infants were $85 \%$ lower than in the previous years [14]. Although the local social distancing index varied between $34.2 \%$ and almost 
$60 \%$ from March to August, schools and daycare centers remained closed throughout this period. All these measures can be associated with these observed and significant lower levels of transmission for both influenza and RSV. But not good enough to prevent the spread of the highly infectious SARS-CoV-2 virus.

There are a few limitations that may be worth addressing. Data refers to only one city in Brazil at only two health care facilities. Also, subjects were not enrolled using a population-based strategy. However, we believe that the large number of patients in the study, its prospective design, and the inclusion of children and adults from diverse environmental and social backgrounds evaluated throughout usual influenza and RSV seasons outweigh issues of external validity from a convenience sample.

The complete absence of detection of influenza and RSV could raise an issue about the quality of our tests and the specimen collection, still we feel confident with our results. GeneXpert@ provides a very automated process, less prone to human processing errors. Moreover, its internal controls (Sample Processing Controls and Probe Check Control) are able to check if there was enough nucleic acid in each sample. We do believe that both influenza and RSV are present in the community, but their numbers are so low that a sample of over 1400 subjects was not able to detect these pathogens.

In summary, our study adds important information regarding the spreading dynamics of high burden respiratory viruses during a period of effective public health measures. The low incidence of RSV and influenza, in contrast with SARS-CoV-2 should be considered in the development of guidelines for antiviral treatment of influenza or in the prevention of RSV with monoclonal antibodies. Moreover, although maintenance such restrictions are not feasible continuously, similar measures could be adopted to control outbreaks due to these viruses. Further, as SARS-CoV-2 prevention depends currently only on non-pharmacological interventions, continuous monitoring of its transmission dynamics is necessary. Hygiene practice and social distancing measures appear to be associated with dramatic reduction of the spread of RSV and influenza.

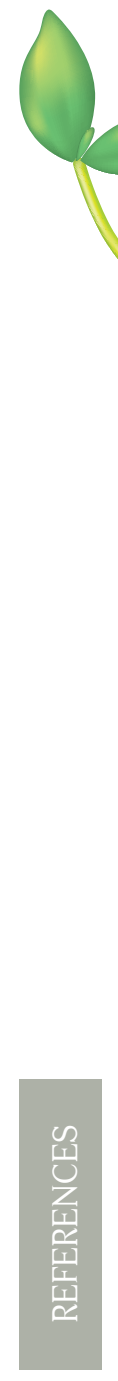

Acknowledgments: We thank the Scientific Committee of the Research Support Center (NAP) of Moinhos de Vento Hospital for technical-scientific consultancy. We thank the nurses and or health works who helped collect patient data, the laboratory team, and the staff from both Hospital Moinhos de Vento and from Hospital Restinga e Extremo Sul. Catia Moreira Guterres, Caroline Cabral Robinson, Aline Andrea da Cunha, Joao Ronaldo Mafalda Krauser, Paulo Sergio Kroeff Schmitz, Marcelo Basso Gazzana, Alexandre Prehn Zavascki, Sidiclei Machado Carvalho, Fabio Jose Rockenbach, Kelly Viegas Antunes, Marcelo da Silva Ferreira, Rafael Garcia Trindade, Thayna Silva Lino, Tiago da Silva Silvano, Adriana da Silva Silveira, Alceu Kuckoski, Ana Paula da Silva Lopes, Andreia Escobar da Costa, Clarice Cardoso Machado, Erica Vieira da Silva, Evelin Inácia da Silva, Luciana Rodrigues Ribeiro, Marcely Mayr da Costa, Morgana Thais Carollo Fernandes, Rafael da Silva Cassafuz, Rita Godoy Soares and Andréa Volkmer, Darleia Radaelli.

COVIDa study group: Camila Dietrich, Caroline Nespolo de David, Luciane Beatriz Kern, Amanda Paz Santos, Tiago Fazolo, Fernando Rovedder Boita, Fernanda Lutz Tolves, Jaina da Costa Pereira, Adriana Isabel Rohden, Glaucia Fragoso Hohenberger, Thainá Dias Luft, Shirlei Villanova Ribeiro, Catia Moreira Guterres, Caroline Cabral Robinson, Débora Vacaro Fogazzi, Eliana Marcia da Ros Wendland, Regis Goulart Rosa, Raul Correia da Silva, Maristênia Machado Araújo, Ana Carolina Monteiro da Rocha, Tássia Rolim Camargo, Giovana Petracco de Miranda, William Jones Dartora, Camila Bonalume Dall'Aqua, Thaís Jacobsen Duarte, Madelaine Correa de Oliveira, Emerson Silveira de Brito, Thayane Martins Dornelles, Ana Paula dos Santos.

Funding: This work was supported by the Ministry of Health of Brazil, through the Institutional Development Program of the Brazilian National Health System (PROAD-SUS) in collaboration with Hospital Moinhos de Vento.

Authorship contributions: All authors listed are responsible for this study and have participated in the concept and design, analysis and interpretation of data, drafting and revising of the manuscript

Competing interests: The authors completed the ICMJE Unified Competing Interest form (available upon request from the corresponding author), and declare no conflicts of interest.

1 Johns Hopkins University. COVID-19 Dashboard by the Center for Systems Science and Engineering (CSSE) at Johns Hopkins University (JHU). 2020 [cited 2020 Sep 9]. Available: https://coronavirus.jhu.edu/map.html. Accessed: 9 September 2020.

2 Lobo A de P, Cardoso-dos-Santos AC, Rocha MS, Pinheiro RS, Bremm JM, Macário EM, et al. COVID-19 epidemic in Brazil: Where are we at? Int J Infect Dis. 2020;97:382-5. Medline:32561425 doi:10.1016/j.ijid.2020.06.044

3 Wiese AD, Everson J, Grijalva CG. Social distancing measures: evidence of interruption of seasonal influenza activity and early lessons of the SARS-CoV-2 pandemic. Clin Infect Dis. 2020. Online ahead of print. Medline:32562538 doi:10.1093/cid/ciaa834

4 Freitas ARR, Donalisio MR. Respiratory syncytial virus seasonality in Brazil: Implications for the immunisation policy for atrisk populations. Mem Inst Oswaldo Cruz. 2016;111:294-30. 
5 Almeida A, Codeço C, Luz PM. Seasonal dynamics of influenza in Brazil: the latitude effect. BMC Infect Dis. 2018;18:695. Medline:30587159 doi:10.1186/s12879-018-3484-z

6 BRASIL, Instituto Brasileiro de Geografia e Estatística. Porto Alegre (RS) | Cidades e Estados | IBGE. IBGE. 2020. Available: https://www.ibge.gov.br/cidades-e-estados/rs/porto-alegre.html. Accessed: 7 February 2021.

7 Cepheid. Xpert® Flu/RSV XC. 2019. Available: www.cepheid.com. Accessed: 10 September 2020.

8 Nolen LD, Seeman S, Bruden D, Klejka J, Desnoyers C, Tiesinga J, et al. Impact of Social Distancing and Travel Restrictions on non-COVID-19 Respiratory Hospital Admissions in Young Children in Rural Alaska. Clin Infect Dis. 2020. Online ahead of print. Medline:32888007

9 Wu D, Lu J, Liu Y, Zhang Z, Luo L. Positive effects of COVID-19 control measures on influenza prevention. Vol. 95. Int J Infect Dis. 2020;95:345-6. Medline:32283283 doi:10.1016/j.jiid.2020.04.009

10 Leuzinger K, Roloff T, Gosert R, Sogaard K, Naegele K, Rentsch K, et al. Epidemiology of SARS-CoV-2 Emergence Amidst Community-Acquired Respiratory 1 Viruses 2. medRxiv. doi:10.1101/2020.07.07.20148163

11 Kim D, Quinn J, Pinsky B, Shah NH, Brown I. Rates of Co-infection between SARS-CoV-2 and Other Respiratory Pathogens. JAMA. 2020;323:2085-6. Medline:32293646 doi:10.1001/jama.2020.6266

12 BRASIL, Ministério da Saúde. Campanha Nacional de Vacinação alcançou 88,8\% do público-alvo. Ministério da Saúde. 2020. Available: https://antigo.saude.gov.br/noticias/agencia-saude/47163-campanha-nacional-de-vacinacao-alcancou-88-8-do-publico-alvo. Accessed: 7 February 2021.

13 BRASIL, Ministério da Saúde, Secretaria de Vigilância em Saúde. 30 Boletim Epidemiológico Especial: Doença pelo Coronavírus COVID-19. 2020. Available: https://ourworldindata.org/coronavirus. Accessed: 21 December 2020.

14 Friedrich F, Ongaratto R, Scotta MC, Veras TN, Stein RT, Lumertz MS, et al. Early Impact of social distancing in response to COVID-19 on hospitalizations for acute bronchiolitis in infants in Brazil. Clin Infect Dis. 2020. Online ahead of print. Medline:32986818 